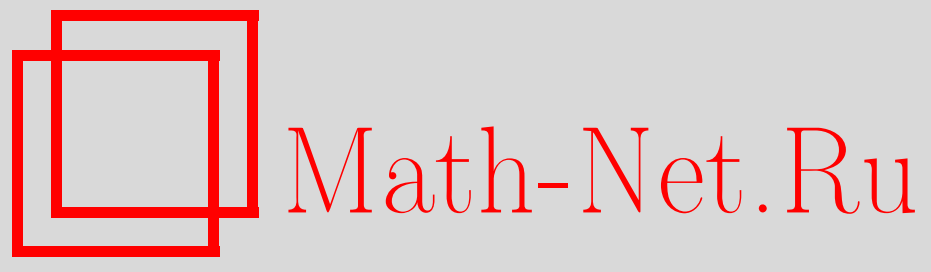

В. Д. Володин, Комбинаторика флаговых симплициальных 3-многогранников, УМН, 2015, том 70, выпуск 1, 181-182

DOI: https://doi.org/10.4213/rm9645

Использование Общероссийского математического портала Math-Net.Ru подразумевает, что вы прочитали и согласны с пользовательским соглашением http://www . mathnet.ru/rus/agreement

Параметры загрузки:

IP : 3.85 .73 .92

26 апреля 2023 г., 18:20:40

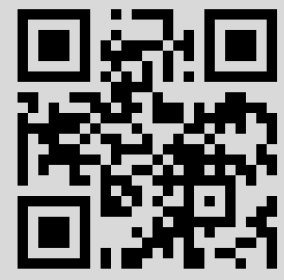




\section{Комбинаторика флаговых симплициальных 3-многогранников}

\section{В. Д. Володин}

Мы будем рассматривать выпуклые симплициальные 3-многогранники. Границы таких многогранников являются симплициальными 2-сферами. Согласно теореме Штейница, верно и обратное утверждение. Поэтому достаточно исследовать комбинаторику симплициальных 2-сфер, забыв об их многогранных реализациях.

Теорема (Э. Штейниц, 1922, см. [3]). Граф Г является графом выпуклого 3-многогранника тогда и только тогда, когда Г является простым, 3-связным и планарным.

ОПредЕлЕниЕ 1. Набор вершин комплекса, не образующий грань, такой, что любое его собственное подмножество образует грань, называется недостающей гранъю комплекса. Комплекс называется флаговым, если все его недостающие грани состоят из двух вершин. Симплициальный многогранник называется флаговым, если его граница является флаговым симплициальным комплексом.

ОПредЕление 2. Стягиванием ребра $e=\left\{v_{1}, v_{2}\right\}$ комплекса $K$ называется операция замены объединения звезд $\mathrm{st}_{K} v_{1} \cup \mathrm{st}_{K} v_{2}$ на звезду $\mathrm{st}_{K} v$ новой вершины $v$ следующим образом: удаляются вершины $v_{1}$ и $v_{2}$ и все содержащие их симплексы, затем добавляются вершина $v$ и такие симплексы $v \sqcup \sigma$, что $v_{1} \sqcup \sigma \in K$ или $v_{2} \sqcup \sigma \in K$. Полученный комплекс обозначается $K / e$.

Теорема 3. Любой флаговый симплициальный 3-многогранник приводится $к$ октаэдру последовательностъю стягиваний ребер так, что на каждом шаге получается флаговый симплициальный 3-многогранник.

В [1] доказано, что комплекс размерности не выше 2 может быть получен из любого своего геометрического подразделения последовательностью стягиваний ребер. Это обобщает результат Штейница-Радемахера [2] о том, что тетраэдр является единственной минимальной (не допускающей стягивания ребра) триангуляцией двумерной сферы. Однако показать, что граница флагового симплициального многогранника является геометрическим подразделением октаэдра, будет недостаточно, поскольку промежуточные многогранники могут не быть флаговыми. Поэтому мы приведем явное доказательство.

Предложение 4. Симплициальная 2-сфера $K$ является флаговой тогда и только тогда, когда для любого ребра е комплекс К/е является симплициальной 2-сферой.

ДокАзАтельство. Пусть $K$ имеет недостающую грань $V,|V|>2$. Если $|V|=4$, то $K$ - граница тетраэдра и стягивание любого ребра дает треугольник. Если $|V|=3$, пусть $V=\left\{v_{1}, v_{2}, v_{3}\right\}$. Стягивая ребро $\left\{v_{1}, v_{2}\right\}$ в вершину $v$, получаем комплекс, не гомеоморфный 2-сфере, поскольку 1-остов полученного комплекса не будет 3-связным (удаление вершин $v$ и $v_{3}$ делает его несвязным).

Пусть комплекс $K /$ е не является многогранным. В силу теоремы Штейница найдутся вершины $v$ и $w$ такие, что комплекс $(K / e) \backslash\{v, w\}$ несвязен. Поскольку исходный комплекс был многогранным, удаление двух вершин не может сделать его несвязным, поэтому можно считать, что вершина $v$ является образом ребра $e=\left\{v_{1}, v_{2}\right\}$ при стягивании. Следовательно, комплекс $K \backslash\left\{v_{1}, v_{2}, w\right\}$ несвязен. Рассмотрим многогранную реализацию комплекса $K$ с вершинами в общем положении. Плоскость $H$, проходящая через вершины $v_{1}, v_{2}$ и $w$, разбивает оставшиеся вершины комплекса $K$ на вершины нижнего и верхнего полупространств. Несложно показать, что любые две вершины нижнего (верхнего) полупространства можно соединить путем, целиком лежащим в нижнем (верхнем) полупространстве. Следовательно, комплекс $K \backslash\left\{v_{1}, v_{2}, w\right\}$ состоит из двух компонент связности, образованных вершинами нижнего и верхнего полупространств. Плоскость $H$ не пересекает ни одного ребра, соединяющего вершины из разных полупространств. Из вышесказанного следует, что вершины $v_{1}, v_{2}$ и $w$ соединены ребрами и образуют недостающую грань комплекса $K$.

Работа выполнена при поддержке РФФИ (грант № 14-01-92612-KО).

DOI: $10.4213 / \mathrm{rm} 9645$ 
Введем частичный порядок на множестве флаговых 2-сфер, полагая $P \preceq Q$, если $P$ получается из $Q$ последовательностью стягиваний ребер. Минимальными в данном случае будут сферы, теряющие флаговость при стягивании любого ребра.

ОПредЕлЕниЕ 5. Будем говорить, что набор вершин $\left\{v_{1}, v_{2}, v_{3}, v_{4}\right\}$ симплициального комплекса образует пояс $\square$, если симплициальный подкомплекс, порожденный этим набором, симплициально изоморфен границе квадрата.

Лемма 6. Пусть $K$ - флаговал симплициальная 2-сфера с ребром е. Симплициальная сфера $K /$ я явлется флаговой тогда и только тогда, когда ребро е не содержится ни в одном поясе сферы $K$.

ДоказАтельство. Покажем, что пояса в $K$, содержащие ребро $е$, являются прообразами недостающих граней мощности 3 в $K / e$ при отображении $K \rightarrow K / e$.

Если ребро $e=\left\{v_{1}, v_{2}\right\}$ содержится в поясе $\left\{v_{1}, v_{2}, v_{3}, v_{4}\right\}$ комплекса $K$, то $K / e$ содержит недостающую грань $\left\{v, v_{3}, v_{4}\right\}$, где $v$ - образ ребра $e$ при стягивании.

Допустим, после стягивания ребра $e=\left\{v_{1}, v_{2}\right\}$ флагового комплекса $K$ в вершину $v$ комплекс $K / e$ не является флаговым. Рассмотрим недостающую грань $V$ комплекса $K / e$ такую, что $|V|>2$. В силу флаговости комплекса $K$ имеем $v \in V$, а комплекс $K / e$ не может быть границей тетраэдра, поэтому $|V|=3$. Пусть $V=\left\{v, v_{3}, v_{4}\right\}$, тогда $v_{1}, v_{2} \in \mathrm{lk}_{K} v_{3} \cup \mathrm{lk}_{K} v_{4}$, но, так как $V$ не образует симплекс в $K / e$, одна из вершин $v_{1}$ и $v_{2}$ содержится в $\mathrm{lk}_{K} v_{3} \backslash \mathrm{lk}_{K} v_{4}$, а другая - в $\mathrm{lk}_{K} v_{4} \backslash \mathrm{lk}_{K} v_{3}$. Поэтому набор $\left\{v_{1}, v_{2}, v_{3}, v_{4}\right\}$ является поясом, содержащим ребро $e$.

Лемма 7. Пусть $K$ - минимальная флаговая симплициальная 2-сфера. Тогда существует вершина $w \in K$, линк которой является поясом в $K$.

ДокАЗАтельство. Рассмотрим произвольную вершину $v \in K$. В силу леммы 6 каждое ребро содержится в некотором поясе. Через $W_{i}^{1}$ и $W_{i}^{2}$ обозначим симплициальные шары, на которые пояс $\square_{i}$ разделяет сферу $K$.

Существует пояс $\square_{0}$, содержащий $v$, такой, что любой пояс $\square^{\prime}$, содержащий $v$ и пересекающий внутренность $W_{0}^{1}$, пересекает и внутренность $W_{0}^{2}$. Действительно, рассмотрим пояс $\square_{1}$, содержащий $v$. Если найдется другой пояс $\square_{2}$, который содержится в $W_{1}^{1}$, то $W_{2}^{1} \subset W_{1}^{1}$. Далее продолжим выбирать $\square_{i}$ так, что $W_{i}^{1} \subset W_{i-1}^{1}$. В какой-то момент выбор следующего пояса станет невозможен, и последний выбранный пояс будет удовлетворять условию выше.

Пусть $v^{\prime} \in \square_{0}$, а $\left\{v, v^{\prime}\right\}$ не является ребром. Каждое ребро $\{v, w\}$, где $w$ - внутренняя вершина $W_{0}^{1}$, содержится в некотором поясе $\square_{v, w}$, пересекающем внутренность $W_{0}^{2}$. Тогда $\square_{0} \cap \square_{v, w}=\left\{v, v^{\prime}\right\}$ в силу 2-связности пояса $\square_{v, w}$. Следовательно, все вершины $W_{0}^{1} \cap \mathrm{lk}_{K} v$ соединены ребром с вершиной $v^{\prime}$. Из выбора множества $W_{0}^{1}$ следует, что $w$ - его единственная внутренняя вершина. Поэтому $\operatorname{lk}_{K} w=\square_{0}$.

ДоКАЗАТЕЛЬСтво теоремы 3. Пусть $K$ - минимальная флаговая симплициальная 2-сфера. Докажем, что $K$ - граница октаэдра. Пусть $w$ - вершина из леммы 7. Рассмотрим пояс $\square_{1}$, содержащий $w$, и пояс $\square_{2}$, содержащий $w$ и вершины из $\mathrm{lk}_{K} w \backslash \square_{1}$. У $\square_{1}$ и $\square_{2}$ есть общая вершина $w$. Поскольку множество $K \backslash \square_{1}$ несвязно, а пояс $\square_{2}$ является 2-связным, имеется и другая общая вершина $v$. Тогда все 4 вершины подкомплекса $\mathrm{lk}_{K} w$ соединены ребрами с вершинами $v$ и $w$. Так как сфера $K$ флаговая, она не имеет других вершин и, следовательно, является границей октаэдра.

\section{Список литературы}

[1] S. Melikhov, "Combinatorics of embeddings", arXiv: 1103.5457. [2] E. Steinitz, H. Rademacher, Vorlesungen über die Theorie der Polyeder unter Einschluss der Elemente der Topologie, Springer, 1976. [3] Г. М. Циглер, Теория многогранников, МЦНМО, М., 2014.

\section{В. Д. Володин (V.D. Volodin)}

Институт проблем передачи информации им. А. А. Харкевича РАН

E-mail: volodinvadim@gmail.com
Представлено В. М. Бухштабером Принято редколлегией 05.12.2014 